\title{
Study of solution stability formulas for certain fractional
}

\author{
differential equations
}

\section{Abdulaziz Rabian saeed ALjuraishi}

\begin{abstract}
The main objective of this work is to study the stability of solutions of a system of linear and nonlinear fractional differential equations. The derivatives considered are within the meaning of Caputo, and of order between 0 and 2.
\end{abstract}

\section{key words}

Caputo fractional derivative, fractional differential equations, Volterra integral equations, Laplace transform. 


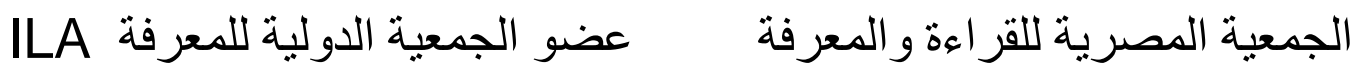




\section{History}

In mathematics, fractional calculus is a branch of analysis that studies the possibility that a differential operator can be raised to a non-integer order. The subject of fractional calculus has gained a considerable popularity during the past three decades, mainly due to its demonstrated applications in many fields of science and engineering. Indeed, it provides several potentially useful tools to solve differential and integral equations, and various other problems involving special functions of mathematical physics, as well as their extensions and generalizations in one and in several variables.

Leibniz in a letter dated September 30, 1695 replied: "It will lead to a paradox, from which one day useful consequences will be drawn". Several authors consider this letter dated September 30, 1695, as the time of birth of the fractional calculation. So fractional calculus is a mathematical subject dating back over 300 years.

The mention of fractional derivatives was made in a certain context, (for example) Euler in 1730, Lagrange in 1772, Laplace in 1812, Lacroix in 1819, Fourier in 1822, Liouville in 1832, Riemann in 1847, Greer in 1859, Holmgren in 1865, Grinwald in 1867, Letnikov in 1868, Sonin in 1869, Laurent in 1884, Nekrassov in 1888, Krug in 1890, and Weyl in 1917. In fact, in his 700-page manual, entitled " Traité du calcul différentiel et du calcul intégral" (second edition; Courcier, Paris, 1819), SF Lacroix devoted two pages (pp. 409-410) to fractional calculus, finally showing that:

$$
\frac{d^{\frac{1}{2}}}{d v^{\frac{1}{2}}} v=\frac{\sqrt[2]{v}}{\sqrt{\pi}}
$$

The first work, devoted exclusively to the subject of fractional calculus, is the book of Oldham and Spanier published in 1974. In fact, many other works (books, edited volumes, and conference proceedings) have also appeared. There exists the remarkably comprehensive encyclopedic-type monograph by Samko, Kilbus and Marichev, which was published in Russian in 1987 and in English in 1993, and the book devoted largely to fractional differential and integral equations by Miller and Ross, which was published in 1993. Today, there are at least two international newspapers that are devoted almost entirely to the object of fractional calculus:

Journal of Fractional Calculus

Fractional Calculus and Applied Analysis 
Recently, the stability theory of fractional differential equations (FDEs) is of main interest in physical systems. Moreover, some stability results have been found [1-9]. These stability results are almost about the linear fractional differential systems with commensurate order [2].

For example, a necessary and sufficient condition on asymptotic stability of linear fractional differential system with order $0<\alpha \leq 1$ was first given in [3]. Then, some literatures on the stability of linear fractional differential systems with order $0<\alpha \leq 1$ have been appeared [4-5]. However, not all the fractional differential systems have fractional orders in $(0,1)$. There exist fractional models which have fractional orders lying in $(1,2)$, for example, super-diffusion [6]. Hence, the stability of linear fractional differential systems with order $0<\alpha<2$ has also been considered by using the conversion methods and transfer function [7-8]. 


\section{Introduction}

Let's Compare the following two systems of equations: $0<\lambda<1 ; 0<\alpha<1$ and $\mathrm{x}(0)=\mathrm{x} 0$.

$$
\begin{aligned}
& \operatorname{Dx}(\mathrm{t})=\lambda \mathrm{t}^{\lambda-1} \quad \rightarrow \quad \mathrm{x}(\mathrm{t})=\mathrm{t}^{\lambda}+\mathrm{x} 0 \\
& { }^{c} \mathrm{D}_{0+}^{\alpha} \mathrm{x}(\mathrm{t})=\lambda \mathrm{t}^{\lambda-1} \quad \rightarrow \mathrm{x}(\mathrm{t})=\frac{\lambda \Gamma(\lambda) t^{\lambda+\alpha-1}}{\Gamma(\lambda+)}+\mathrm{x} 0
\end{aligned}
$$

Or ${ }^{c} D_{0+}^{\alpha}$ is the Caputo derivative defined by (11)

We have indeed observed, that the whole order system $\left(^{*}\right)$ is unstable whatever $\lambda \in$ ] $0,1\left[\right.$. The non-integer or fractional order system $\left(^{* *}\right)$ is stable whatever $\left.\lambda \in\right] 0,1-\alpha[$. This generally shows that fractional systems have different characteristics compared to whole order systems.

The aim of this article is to study the solution stability formulas for certain fractional differential equations. It consists of three parts:

Part 1: Fundamental notions

Part 2: The stability of linear fractional differential systems of order $\alpha \in(0 ; 2)$

Part 3: The stability of nonlinear fractional differential systems of order $\alpha$ $\in(0 ; 2)$ 


\section{Fundamental notions}

The aim of this part is to present, in a synthetic and unified manner, the elements on the theory of fractional calculus and systems of non-integer derivatives on which our work described in parts 2 and 3 is based.

\subsection{Required functions for fractions calculator}

In this part, we present the functions: Euler's Gamma function, Beta function and Mittag-Leffler function which will be used in other parts. These functions play a very important role in the theory of fractional calculus.

\subsubsection{Gamma function}

One of the basic functions of fractional calculus in mathematics is the Euler's Gamma function (or Gamma function). It is a complex function which extends the factorial function to the set of complex numbers.

\section{Definition}

The Gamma function is defined by the following integral:

$$
\Gamma(\mathrm{z})=\int_{0}^{\infty} \mathrm{t}^{\mathrm{z}-1} \mathrm{e}^{-\mathrm{t}} \mathrm{dt} \quad(\operatorname{Re}(\mathrm{z})>0)
$$

For all complex numbers $\mathrm{z}$, the following recurrence relation is true:

$$
\Gamma(\mathrm{z}+1)=z \Gamma(\mathrm{z})
$$

Consequently, for positive integers:

$$
\Gamma(\mathrm{n}+1)=\mathrm{n} !
$$




\subsubsection{Beta function}

\section{Definition}

The beta function is defined by the Euler integral:

$$
\mathrm{B}(\mathrm{z}, \mathrm{w})=\int_{0}^{1} \mathrm{t}^{\mathrm{z}-1}(1-t)^{w-1} \mathrm{dt}(\operatorname{Re}(\mathrm{z})>0, \operatorname{Re}(\mathrm{w})>0)
$$

This function is linked to the gamma function by the relation:

$$
\mathrm{B}(\mathrm{z}, \mathrm{w})=\frac{\Gamma(\mathrm{z}) \Gamma(\mathrm{w})}{\Gamma(\mathrm{z}+\mathrm{w})} \quad\left(\mathrm{z}, \mathrm{w} \notin \mathrm{Z}_{0}^{-}\right)
$$

\subsubsection{Mittag-Leffler function}

The exponential function $\mathrm{e}^{\mathrm{z}}$, plays a very important role in the theory of integerorder differential equations. The generalization of the exponential function with a single parameter was introduced by G.M. Mittag-Leffler.

\section{Definition}

The standard definition of Mittag-Leffler is given as follows:

$$
\mathrm{E}_{\alpha}(\mathrm{z})=\sum_{\mathrm{k}=0}^{+\infty} \frac{\mathrm{z}^{\mathrm{k}}}{\Gamma(\alpha \mathrm{k}+1)} \quad(\operatorname{Re}(\alpha)>0, \mathrm{z} \in \mathrm{C})
$$

A two-parameter function of the M-L (Mittag-Leffler) type is defined by the series expansion.

$$
E_{\alpha, \beta}(z)=\sum_{k=0}^{+\infty} \frac{z^{k}}{\Gamma(\alpha \mathrm{k}+\beta)} \quad(\operatorname{Re}(\alpha)>0, \beta, \mathrm{z} \in \mathrm{C})
$$




\subsection{Riemann Liouville fractional integrals and derivatives}

In this section we give the definitions of Riemann-Liouville fractional integrals and derivatives on a bounded interval on $\mathrm{R}$.

We can start by examining a (unique) formula which gives successive primitives of a continuous function for example

Let $\mathrm{y}:[\mathrm{a} ; \mathrm{b}) \rightarrow \mathrm{R},(\mathrm{b} \leq+\infty)$ a continuous function; a primitive of $\mathrm{y}$ which cancels out at a is given by:

$$
\left(I_{\alpha}^{1} y\right)(x)=\int_{\alpha}^{x} y(t) d t
$$

For a second primitive we will have

$$
\left(I_{\alpha}^{2} y\right)(x)=\int_{\alpha}^{x}\left(\int_{\alpha}^{s} y(t) d t\right) d s
$$

Fubini's theorem brings us back this double integral to a simple integral

$$
\left(I_{\alpha}^{2} y\right)(x)=\int_{\alpha}^{x}(x-t) y(t) d t
$$

Then an iteration gives

$$
\left(I_{\alpha}^{\mathrm{n}} \mathrm{y}\right)(\mathrm{x})=\int_{\alpha}^{\mathrm{x}} \frac{(\mathrm{x}-\mathrm{t})^{\mathrm{n}-1}}{(\mathrm{n}-1) !} \mathrm{y}(\mathrm{t}) \mathrm{dt}
$$




\subsubsection{Riemann-Liouville fractional integral}

\section{Definition}

Let $\Omega=(\mathrm{a} ; \mathrm{b}](-\infty<\mathrm{a}<\mathrm{b}<\infty)$ a finite interval of the real axis $\mathrm{R}$. The left and right Riemann-Liouville fractional integrals $\mathrm{I}_{\alpha+}^{\alpha} \mathrm{y}$ and $\mathrm{I}_{\mathrm{b}-\mathrm{y}}^{\alpha}$ of order $\alpha \in \mathrm{C}(\operatorname{Re}(\alpha)>0)$ are defined respectively by:

$$
\begin{aligned}
& \left(I_{\alpha+}^{\alpha} y\right)(x)=\frac{1}{\Gamma(\alpha)} \int_{\alpha}^{x} \frac{y(t) d t}{(x-t)^{1-\alpha}}(x>\alpha, \operatorname{Re}(\alpha)>0) \\
& \left(I_{b-}^{\alpha} y\right)(x)=\frac{1}{\Gamma(\alpha)} \int_{x}^{b} \frac{y(t) d t}{(t-x)^{1-\alpha}}(x<b, \operatorname{Re}(\alpha)>0)
\end{aligned}
$$

Here $\Gamma(\alpha)$ denotes the Euler's gamma function.

\subsubsection{Riemann-Liouville fractional derivative}

The left and right Riemann-Liouville fractional derivatives $\mathrm{D}_{\alpha+}^{\alpha} \mathrm{y}$ and $\mathrm{D}_{\mathrm{b}-\mathrm{y}}^{\alpha}$ of order $\alpha \in \mathrm{C}(\operatorname{Re}(\alpha)>0)$ are defined respectively by:

$$
\begin{aligned}
& \left(\mathrm{D}_{\alpha+}^{\alpha} \mathrm{y}\right)(\mathrm{x})=\left(\frac{d}{d x}\right)^{n}\left(\mathrm{I}_{\alpha+}^{\mathrm{n}-\alpha} \mathrm{y}\right)(\mathrm{x}) \\
& =\frac{1}{\Gamma(\mathrm{n}-\alpha)}\left(\frac{d}{d x}\right)^{n} \int_{\alpha}^{\mathrm{x}} \frac{\mathrm{y}(\mathrm{t}) \mathrm{dt}}{(\mathrm{x}-\mathrm{t})^{\alpha-\mathrm{n}+1}} \quad(\mathrm{x}>\alpha ; \mathrm{n}=[\operatorname{Re}(\alpha)]+1)
\end{aligned}
$$

And

$$
\begin{aligned}
\left(\mathrm{D}_{\mathrm{b}-}^{\alpha} \mathrm{y}\right)(\mathrm{x}) & =\left(-\frac{d}{d x}\right)^{n}\left(\mathrm{I}_{\mathrm{b}-}^{\mathrm{n}-\alpha} \mathrm{y}\right)(\mathrm{x}) \\
& =\frac{1}{\Gamma(\mathrm{n}-\alpha)}\left(-\frac{d}{d x}\right)^{n} \int_{\mathrm{x}}^{\mathrm{b}} \frac{\mathrm{y}(\mathrm{t}) \mathrm{dt}}{(\mathrm{t}-\mathrm{x})^{\alpha-\mathrm{n}+1}}(\mathrm{x}<\mathrm{b} ; \mathrm{n}=[\operatorname{Re}(\alpha)]+1)
\end{aligned}
$$

$[\operatorname{Re}(\alpha)]$ is the real number of $\operatorname{Re}(\alpha)$. 


\subsection{Caputo Fractional Derivative}

Let $[a, b]$ : a finite interval of the real axis $R, D_{\alpha+}^{\alpha}[y(t)](x)=\left(D_{\alpha+}^{\alpha} y\right)(x)$ and , $\mathrm{D}_{\mathrm{b}-}^{\alpha}[\mathrm{y}(\mathrm{t})](\mathrm{x})=\left(\mathrm{D}_{\mathrm{b}-\mathrm{y}}^{\alpha}\right)(\mathrm{x})$ the Riemann-Liouville fractional derivatives of order $\alpha \in \mathrm{C}$ $(\operatorname{Re}(\alpha) \geq 0)$ defined respectively by (10) and (11). The fractional derivatives ${ }^{c} D_{\alpha+}^{\alpha}(\mathrm{x})$ and ${ }^{c} D_{b-}^{\alpha}(x)$ of order $\alpha \in C(\operatorname{Re}(\alpha) \geq 0)$ over the interval [a; b] are defined respectively by:

$$
\left({ }^{(} D_{\alpha+}^{\alpha} y\right)(x)=\left(D_{\alpha+}^{\alpha}\left[y(t)-\sum_{k=0}^{n-1} \frac{y^{(k)}(\alpha)}{k !}(t-\alpha)^{k}\right]\right)(x)
$$

And

$$
\left({ }^{c} D_{b-}^{\alpha} y\right)(x)=\left(D_{b-}^{\alpha}\left[y(t)-\sum_{k=0}^{n-1} \frac{y^{(k)}(\alpha)}{k !}(b-t)^{k}\right]\right)(x)
$$

With: $\mathrm{n}=[\operatorname{Re}(\alpha)]+1$ for $\alpha \notin \mathrm{N}_{0}, \mathrm{n}=\alpha$ for $\alpha \in \mathrm{N}_{0}$

The derivatives ( $\left.{ }^{c} \mathrm{D}_{\alpha+\mathrm{y}}^{\alpha} \mathrm{y}\right)(\mathrm{x})$ and $\left({ }^{(} \mathrm{D}_{\mathrm{b}-\mathrm{y}}^{\alpha}\right)(\mathrm{x})$ are called the left, right Caputo fractional derivative of order $\alpha$.

\subsection{Laplace transformation}

The Laplace transformation belongs to the very large family of integral transformations, which establish a relationship between a function $f$ and its transform $F$ in the form:

$$
F(w)=\int_{I} k(w, t) f(t) d t
$$

\section{Definition}

Let $f(t)$ be defined for $t \geq 0$. The Laplace transform of $f(t)$, denoted by $F(z)$ or $\mathrm{L}\{\mathrm{f}(\mathrm{t})\}$, is an integral transform given by the Laplace integral:

$$
L(f)(z)=\int_{\mathrm{R}^{+}} \mathrm{f}(\mathrm{t}) \mathrm{e}^{-\mathrm{zt}} \mathrm{dt}
$$




\section{The stability of linear fractional differential systems of order $\alpha \in(0 ; 2)$}

\subsection{Preliminaries}

It is useful to recall some lemmas and the asymptotic formulas of the Mettag-leffler function.

\section{Lemma 2.1.1 (Jordan Canonical Form)}

Let $\mathrm{A}$ be a $\mathrm{n}^{*} \mathrm{n}$ complex matrix (i.e., entries of $\mathrm{A}$ could be either real numbers or complex numbers). Then there are a complex invertible matrix $\mathrm{P}$ and a complex block diagonal matrix J such that

$$
\mathrm{P}^{-1} \mathrm{AP}=\mathrm{J}
$$

Where J is a Jordan block (i.e. a square matrix which has zero entries everywhere except on the diagonal, where the entries are a fixed scalar, and except on the super diagonal, where the entries are either all 0 or all 1 ).

Lemma 2.1.2

If $0<\alpha<2, \beta$ an arbitrary complex number and $\mu$ is an arbitrary real number, such as:

$$
\frac{\alpha \pi}{2}<\mu<\min \{\pi, \pi \alpha\}
$$

\section{Lemma 2.1.3}

If $\mathrm{A} \in \mathrm{C}^{\mathrm{n} * \mathrm{n}}$ and $0<\alpha<2, \beta$ is an arbitrary real number, $\mu$ satisfied $\frac{\alpha \pi}{2}<\mu<\min \{\pi, \pi \alpha\}$ and $\mathrm{C}>0$, real constants then:

$$
\left\|E_{\alpha, \beta}(\mathrm{A})\right\| \leq \frac{C}{1+\|A\|}
$$

$\operatorname{Or} \mu \leq|\operatorname{Arg}(\lambda)| \leq \pi, \forall \lambda \in$ spec (A) such as spec (A): the eigenvalues of the matrix $A$ and $\|$.$\| are called l_{2}$-norm. 
Lemma 2.1.4

$$
\text { If } \mathrm{x}(\mathrm{t}) \leq \mathrm{h}(\mathrm{t})+\int_{t_{0}}^{t} k(s) x(s) d s \quad \mathrm{t} \in\left[t_{0}, \mathrm{~T}\right)
$$

Where all the functions involved are continuous on $\left[t_{0}, \mathrm{~T}\right), \mathrm{T} \leq+\infty$ and $\mathrm{k}(\mathrm{x}) \geq 0$, then $\mathrm{x}(\mathrm{t})$ satisfied.

$$
\mathrm{x}(\mathrm{t}) \leq \mathrm{h}(\mathrm{t})+\int_{t_{0}}^{t} k(s) h(s) \exp \left[\int_{s}^{t} k(u) d u\right] d s \quad \mathrm{t} \in\left[t_{0}, \mathrm{~T}\right)
$$

\subsection{Definitions on stability}

\section{Definition 2.2.1}

The constant $\mathrm{x}$ e $\mathrm{q}$ is an equilibrium point of fractional differential system $D_{t_{0}, t}^{\alpha} x(t)=f(t, x)$ if and only if $f\left(t, x_{e q}\right)=\left.D_{t_{0}, t}^{\alpha} x(t)\right|_{x(t)=x_{e q}}$ for all $t>t 0$, or the operator $D_{t_{0}, t}$ is $\mathrm{CD}_{\mathrm{t}_{0}, \mathrm{t}}^{\alpha}$ or ${ }_{\mathrm{R} \cdot \mathrm{L}} \mathrm{D}_{\mathrm{t}_{0}, \mathrm{t}}^{\alpha}$.

Without loss of generality, Suppose the trivial equilibrium point $x_{e q}=0$, we introduce the following definitions.

\section{Definition 2.2.2}

The zero solution of $\mathrm{D}_{\mathrm{t}_{0}, \mathrm{t}}^{\alpha} \mathrm{x}(\mathrm{t})=\mathrm{f}(\mathrm{t}, \mathrm{x})$ of order $0<\alpha<1[$ or $1<\alpha<2]$ is said to be stable if, for any initial value $x_{0}$ [or $x_{k}(\mathrm{k}=0,1)$ ], it exists $\varepsilon>0$ such as $\|\mathrm{x}(\mathrm{t})\| \leq \varepsilon$ for all $\mathrm{t}$ $>t_{0}$.

\section{Definition 2.2.3}

The zero solution is said to be asymptotically stable if in addition to be stable, $\|\mathrm{x}(\mathrm{t})\| \rightarrow 0$ when $\mathrm{t} \rightarrow+\infty$. 


\subsection{The stability of linear fractional differential systems of order $\alpha \in$} $(0 ; 1)$

\subsection{1. autonomous linear fractional differential system}

In this section we study the stability of the solution of the autonomous linear fractional differential system with the Caputo derivative in the form:

$$
\left\{\begin{array}{l}
\frac{d^{\alpha} x}{d t^{\alpha}}=\operatorname{Ax}(\mathrm{t}) \\
\mathrm{x}\left(\mathrm{t}_{0}\right)=\mathrm{x}_{0}
\end{array} \quad \mathrm{t}>t_{0}\right.
$$

Or $\mathrm{x} \in \mathrm{R}^{\mathrm{n}}$, the matrix $\mathrm{A} \in \mathrm{R}^{\mathrm{n} * \mathrm{n}}$ and $0<\alpha<1$

\section{Theorem 2.3.1.1}

The autonomous linear fractional differential system (20) with the Caputo derivative is asymptotically stable if and only if $\left|\operatorname{Arg}\left(\lambda_{1}\right)\right|>\frac{\alpha \pi}{2} \forall \lambda_{1} \in \operatorname{spec}(A)$.

\section{Conclusion}

If $\exists l \in\{1, \ldots, s\}:\left|\operatorname{Arg}\left(\lambda_{1}\right)\right|<\frac{\alpha \pi}{2}$; then the solution to a system (20) is not stable. If the matrix A has one eigenvalue, the solution to a system (20) is not stable.

- If the matrix A has one critical eigenvalue $\lambda_{1}$, that satisfies, $\left|\operatorname{Arg}\left(\lambda_{1}\right)\right|<\frac{\alpha \pi}{2}$ and that the algebraic multiplicity of critical eigenvalue $\lambda_{1}$ is not equal to the geometric multiplicity, so the solution to a system (20) is not stable.

If $1 \in\{1, \ldots, s\}:\left|\operatorname{Arg}\left(\lambda_{1}\right)\right| \geq \frac{\alpha \pi}{2}$ such critical eigenvalues that satisfy $\left|\operatorname{Arg}\left(\lambda_{1}\right)\right|=\frac{\alpha \pi}{2}$ have the same algebraic and geometric multiplicity, so the solution to a system (20) is stable, without having asymptotic stability.

\subsubsection{Non-autonomous linear fractional differential system}

In this section we study the stability of the solution of the non-autonomous linear fractional differential system with the Caputo derivative in the form:

$$
\left\{\begin{array}{l}
\frac{d^{\alpha} x}{d t^{\alpha}}=\mathrm{Ax}(\mathrm{t})+\mathrm{B}(\mathrm{t}) \mathrm{x}(\mathrm{t}) \quad \mathrm{t}>t_{0} \\
\mathrm{x}\left(\mathrm{t}_{0}\right)=\mathrm{x}_{0}
\end{array}\right.
$$

Or $\mathrm{x} \in \mathrm{R}^{\mathrm{n}}$, the matrix $A \in \mathrm{R}^{\mathrm{n} * \mathrm{n}}, 0<\alpha<1$ and $\mathrm{B}(\mathrm{t}):\left[\mathrm{t}_{0},+\infty\left[\rightarrow \mathrm{R}^{\mathrm{n} * \mathrm{n}}\right.\right.$ is a continuously differentiable matrix. 


\section{Theorem 2.3.2.1}

If $\forall \lambda_{1} \in \operatorname{spec}(A) \neq 0,\left|\operatorname{Arg}\left(\lambda_{1}\right)\right| \geq \frac{\alpha \pi}{2}$, such critical eigenvalues that satisfy $\left|\operatorname{Arg}\left(\lambda_{1}\right)\right|=$ $\frac{\alpha \pi}{2}$ have the same algebraic and geometric multiplicity and $\int_{t_{0}}^{\infty}\|B(t)\|$ is bounded. So the solution to a system (21) is stable.

We get the solution to a system (21) using the Laplace Transform and the Inverse Laplace Transform:

$$
x(t)=E_{\alpha}\left(A\left(t-t_{0}\right)^{\alpha}\right) x_{0}+\int_{t_{0}}^{t}(t-T)^{\alpha-1} E_{\alpha, \alpha}\left(A(t-T)^{\alpha}\right) B(T) x(T) d T
$$

\section{Theorem 2.3.2.2}

If the matrix A satisfy $\forall \lambda_{1} \in \operatorname{spec}(\mathrm{A}) \neq 0,\left|\operatorname{Arg}\left(\lambda_{1}\right)\right|>\frac{\alpha \pi}{2}$ and $\| \mathrm{B}(\mathrm{t})||=\mathrm{O}\left(t-t_{0}\right)^{\gamma}(-$ $1<\gamma<1-\alpha, t_{0}>0$ ) for $\mathrm{t} \geq 0$.

So: the solution to a system (21) is asymptotically stable.

\subsubsection{Disturbed linear fractional differential system}

In this section we study the stability of the disturbed linear fractional differential system with the Caputo derivative in the form:

$$
\begin{cases}\frac{d^{\alpha} x}{d t^{\alpha}}=\mathrm{Ax}(\mathrm{t})+\mathrm{f}(\mathrm{t}, \mathrm{x}(\mathrm{t})) & \mathrm{t}>t_{0} \\ x^{(k)}\left(\mathrm{t}_{0}\right)=\mathrm{x}_{\mathrm{k}} & (\mathrm{k}=0 ; 1)\end{cases}
$$

Or $x \in R n$, the matrix $A \in \mathrm{R}^{\mathrm{n} * \mathrm{n}}, 1<\alpha<2$ and $\mathrm{f}(\mathrm{t}, \mathrm{x}(\mathrm{t})):\left[\mathrm{t}_{0},+\infty\left[{ }^{*} \mathrm{R}^{\mathrm{n}} \rightarrow \mathrm{R}^{\mathrm{n}}\right.\right.$ is a continuous function with $f(t, 0)=0, f(t, x)$ satisfies the Lipschtz condition for $x$, the unique solution to a system (23) is:

$\mathrm{x}(\mathrm{t})=\sum_{k=0}^{1}\left(t-t_{0}\right)^{k} \mathrm{E}_{\alpha, \mathrm{k}+1}\left(\mathrm{~A}\left(\mathrm{t}-\mathrm{t}_{0}\right)^{\alpha}\right) \mathrm{x}_{\mathrm{k}}+\int_{\mathrm{t}_{0}}^{\mathrm{t}}(\mathrm{t}-\mathrm{T})^{\alpha-1} \mathrm{E}_{\alpha, \alpha}\left(\mathrm{A}(\mathrm{t}-\mathrm{T})^{\alpha}\right) \mathrm{f}(\mathrm{t}, \mathrm{x}(\mathrm{T})) \mathrm{dt}$ (24) 


\section{Theorem 2.3.3.1}

If the matrix A satisfy $\forall \lambda_{1} \in \operatorname{spec}(A) \neq 0,\left|\operatorname{Arg}\left(\lambda_{1}\right)\right| \geq \frac{\alpha \pi}{2}$ such critical eigenvalues that satisfy $\left|\operatorname{Arg}\left(\lambda_{1}\right)\right|=\frac{\alpha \pi}{2}$ have the same algebraic and geometric multiplicity, moreover there is a function $\gamma(t)$ that satisfy the following conditions:

$$
\begin{aligned}
& * \int_{\mathrm{t}_{0}}^{\infty} \gamma(t) d t \text { is bounded } \\
& \text { * }\|\mathrm{f}(\mathrm{t}, \mathrm{x})\| \leq \gamma(t)\|\mathrm{x}(\mathrm{t})\|
\end{aligned}
$$

So: the solution to a system (23) is stable.

\section{Conclusion}

Consider the following linear fractional system of order $\alpha \in(0 ; 1)$

$$
\left\{\begin{array}{l}
\frac{d^{\alpha} x}{d t^{\alpha}}=\mathrm{Ax}(\mathrm{t}) \\
\mathrm{x}\left(\mathrm{t}_{0}\right)=\mathrm{x}_{0} \quad \mathrm{t}>t_{0}
\end{array}\right.
$$

Or $\mathrm{x} \in \mathrm{R}^{\mathrm{n}}$, the matrix $A \in \mathrm{R}^{\mathrm{n} * \mathrm{n}}, 0<\alpha<1$

The stability theorem (2.3.1.1), it determines the different stable and unstable regions (Figure 1)

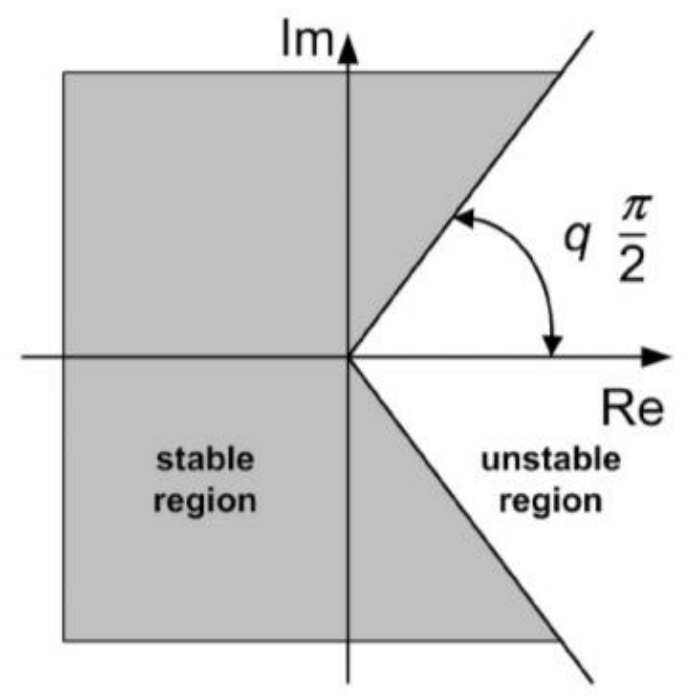

Figure 1: Stability regions of linear fractional differential systems of order $\alpha \in(0 ; 1)$ 
Consider the following linear fractional system of order $\alpha \in(1 ; 2)$

$$
\left\{\begin{array}{l}
\frac{d^{\alpha} x}{d t^{\alpha}}=\mathrm{Ax}(\mathrm{t}) \quad \mathrm{t}>t_{0} \\
x^{(k)}\left(\mathrm{t}_{0}\right)=\mathrm{x}_{\mathrm{k}}
\end{array} \quad(\mathrm{k}=0 ; 1)\right.
$$

Or $\mathrm{x} \in \mathrm{R}^{\mathrm{n}}$, the matrix $A \in \mathrm{R}^{\mathrm{n} * \mathrm{n}}, 1<\alpha<2$

The stability theorem (2.3.2.1), it determines the different stable and unstable regions (Figure 2)

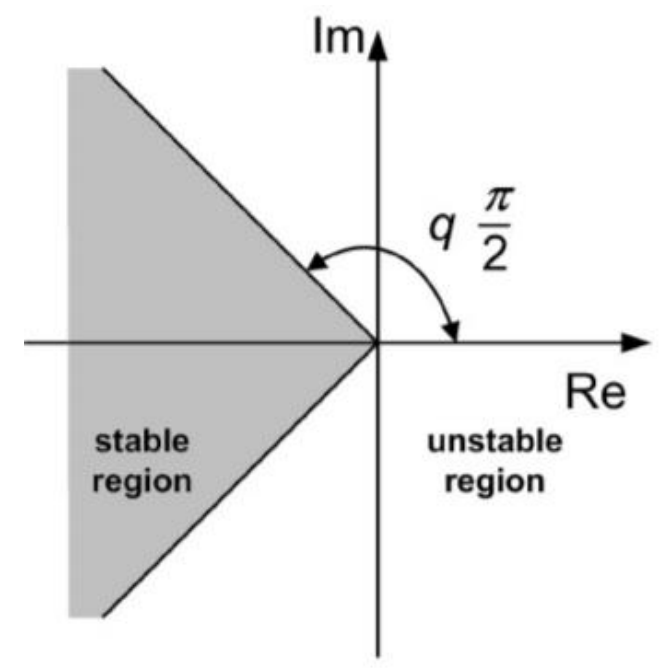

Figure 2: Stability regions of linear fractional differential systems of order $\alpha \in$ 


\section{The stability of nonlinear fractional differential systems of order $\alpha \in(0 ; 2)$}

\subsection{Definitions}

\section{Definition 3.1.1}

If there exist a constant e such that $f(e)=0$, so " $e$ " is said: the point of equilibrium for $\frac{d^{\alpha} x}{d t^{\alpha}}=f(x)$

\section{Definition 3.1.2}

The point of equilibrium " $\mathrm{e}$ " is said:

Locally stable: if and only if $\forall \varepsilon>0, \exists \sigma>0$ such as $|\mathrm{x}(\mathrm{t})-\mathrm{e}|<\varepsilon$ for $\forall x_{0} \in\{\mathrm{z}$ : $|\mathrm{z}-\mathrm{e}|<$ $\sigma\}$ and $\forall \mathrm{t} \geq 0$

* locally asymptotically stable: if and only if the point of equilibrium is locally stable and $\lim _{t \rightarrow+\infty} x(t)=\mathrm{e}$

\subsection{The stability of nonlinear fractional differential systems of order $\alpha$ $\in(0 ; 1)$}

In this section we study the local asymptotic stability of the point of equilibrium of the nonlinear fractional differential system in the form:

$$
\left\{\begin{array}{l}
\frac{d^{\alpha} x}{d t^{\alpha}}=f(x) \\
x(0)=x_{0}
\end{array} \quad t>0\right.
$$

Or $\mathrm{f}$ is continuous, $0<\alpha<1$ and the derivative is in the sense of Caputo derivative. 


\subsection{The stability of nonlinear fractional differential systems of order $\alpha$ $\in(1 ; 2)$}

In this section we study the local asymptotic stability of the solution of the nonlinear fractional differential system in the form:

$$
\begin{cases}\frac{d^{\alpha} x}{d t^{\alpha}}=f(x) & t>0 \\ x(0)=\alpha & x^{\prime}(0)=b\end{cases}
$$

Or $1<\alpha<2$ and Caputo derivative.

\section{Definition 3.3.1}

Let $\alpha \geq 0$, the operator $\mathrm{I}_{\alpha}^{\alpha}$ defined in $\mathrm{L}_{1}[\mathrm{a}, \mathrm{b}]$ with :

$$
I_{\alpha}^{\alpha} f(x)=\frac{1}{\Gamma(\alpha)} \int_{\alpha}^{x}(x-t)^{\alpha-1} f(t) d t
$$

For $\mathrm{a} \leq \mathrm{x} \leq \mathrm{b}$, is called the integral operator of Riemann-Liouville of order $\alpha$ Note

$$
D^{1} \mathrm{I}_{\alpha}^{1} \mathrm{f}(\mathrm{x})=\frac{d}{d x}\left[\frac{1}{\Gamma(\alpha)} \int_{\alpha}^{\mathrm{x}} \mathrm{f}(\mathrm{t}) \mathrm{dt}\right]=f(x)
$$

\section{Definition 3.3.2}

Let $\alpha \geq 0$, and $n=[\alpha]$ so we define the Caputo fractional derivative of order $\alpha$ with:

$$
{ }^{c} D_{\alpha}^{\alpha} f=I_{\alpha}^{n-1} D^{n} f
$$

with $D^{n} f \in L_{1}[a, b]$

If $\alpha \in N_{0}$, so $n=\alpha:{ }^{c} D_{\alpha}^{\alpha} f=I_{\alpha}^{0} D^{n_{f}}=D^{n_{f}}$ 


\section{Conclusion}

The solution for system of linear fractional differential equations of order $0<\alpha<2$ is stable if and only if all the eigenvalues of the matrix of A satisfy $\left|\operatorname{Arg}\left(\lambda_{1}\right)\right| \geq \frac{\alpha \pi}{2}$ such critical eigenvalues that satisfy $\left|\operatorname{Arg}\left(\lambda_{1}\right)\right|=\frac{\alpha \pi}{2}$ have the same algebraic and geometric multiplicity, without having asymptotic stability.

In the case of scalar equation, after the linearization of linear fractional differential equations of order $0<\alpha<1$, we prove if a function $\mathrm{f}(\mathrm{x})$ is continuous and $\frac{\partial f(0)}{\partial \mathrm{x}}<0$, so the solution is locally asymptotically stable.

- Studying the stability of nonlinear fractional differential equations of order $1<\alpha<$ 2, we transform it into a nonlinear fractional differential equation of order $0<\alpha<$ 1. So, the only question that remains is: how to determine the conditions in the Nonlinear Part, which allow the stability study to a system of linear fractional differential equations. 


\section{BIBLIOGRAPHY}

[1] A. A. Kilbas, H. M. Sprivastava, and J. J. Trujillo, Theory and Applications of Fractional

Differential Equations, Elsevier, San Diego, Calif, USA, 2006.

[2] C. P. Li and F. R. Zhang, .A survey on thestability of fractional differential equations,. The EuropeanPhysical Journal Special Topics, vol. 193, pp. 27.47, 2011.

[3] D. Matignon, Reprsentations en variables d.tat demodles de guides d'ondes avec derivation fractionnaire,These de Doctorat,University Paris-Sud 11, 1994.

[4] D. Matignon, Stability results for fractional differential equations with applications to control processing,.in Proceedings of the IMACS-SMC, vol. 2, pp. 963.968, 1996.

[5] M. Moze, J. Sabatier, and A. Oustaloup, .LMI characterization of fractional systems stability,. in Advances in Fractional Calculus, J. Sabatier, O. P. Agrawal, and J. A. Tenreiro Machado, Eds., pp. 419.434, Springer, Dordrecht, The Netherlands, 2007.

[6] J. Sabatier,M.Moze, and C. Farges, .LMI stability conditions for fractional order systems,.Computer \& Mathematics with Applications, vol. 59, no. 5, pp. 1594.1609, 2010.

[7] C. P. Li and Z. G. Zhao, .Numerical approximation of nonlinear fractional differential equations with subdiffusion and Ssperdiffusion,.Computers and Mathematics with Applications. In press.

[8] R. Malti, O. Cois, M. Aoun, F. Levron, and A. Oustaloup, .Computing impulse response energy of fractional transfer function,.in Proceedings of the 15th IFACWorld Congress, Barcelona, Spain, 2002.

[9] M. S. Tavazoei and M. Haeri, .A note on the stability of fractional order systems,. Mathematics and Computers in Simulation, vol. 79, no. 5, pp. 1566.1576, 2009. 